\title{
Aneurismas paraclinoideos: experiencia personal de 1 año en el Hospital Regional de Temuco Paraclinoid aneurysm: 1 year personal experience at Hospital Regional de Temuco
}

\author{
(Trabajo de ingreso a la Sociedad Chilena de Neurocirugía)
}

\author{
Oscar Jiménez Palma ${ }^{1}$ \\ 1 Neurocirujano, Hospital Regional de Temuco.
}

Rev. Chil. Neurocirugía 43: 102-110, 2017

\begin{abstract}
Resumen
Se presenta la experiencia personal en el tratamiento de 5 pacientes con 7 aneurismas paraclinoideos tratados quirúrgicamente en el Hospital Regional Temuco durante junio de 2015 y julio de 2016 (13 meses). Todos los pacientes fueron previamente discutidos con neurorradiologo Intervencional local y considerados no favorables para terapia endovascular. En todos ellos se realizó una craneotomía mini pterional con clinoidectomía extradural y exposición de la arteria carótida interna cervical. Cuatro pacientes consultaron con hemorragia subaracnoidea y requirieron cirugía cerebral de urgencia. En 6 aneurismas se realizó clipaje y en 1 trapping. Cuatro pacientes no tuvieron deterioro neurológico y evolucionaron favorablemente mientras que 1 paciente falleció por hipoperfusión secundario al trapping de la carótida supraclinoidea. Se enfatiza el manejo interdisciplinario, el plan preoperatorio y el conocimiento de la neuroanatomía en el tratamiento de esta patología.
\end{abstract}

Palabras clave: Aneurisma paraclinoideo, aneurisma oftálmico, clinoidectomía extradural, mini pterional.

\begin{abstract}
A personal experience is presented in treating 5 patients with 7 paraclinoid aneurysm who underwent surgery at Hospital Regional Temuco between june 2015 and july 2016 (13 months). All patients were previously discussed with local interventional neuroradiologist considering them not favorable to endovascular therapy. Mini pterional craniotomy with extradural clinoidectomy and internal cervical carotid artery exposure was performed in all of them. 4 patients presented with subarachnoid hemorrhage and required urgent brain surgery. Direct clipping was optimal in 6 aneurysm and 1 was treated with trapping. 4 patients had no neurological deteriotation with excellent outcome and 1 patient died because of hypoperfusion secondary to the supraclinoid carotid trapping. Interdisciplinary management, preoperative planning and neuroanatomy knowledge are emphasized in order to treat this pathology.
\end{abstract}

Key words: Paraclinoid aneurysm, ophthalmic aneurysms, extradural clinoidectomy, mini pterional.

\section{Introducción}

Un aneurisma cerebral sacular es una debilidad en la pared del vaso que lleva a una dilatación focal con riesgo de sangrado y a lesiones secundarias a la hemorragia subaracnoídea con alto riesgo de morbilidad o muerte ${ }^{1}$. Son lesiones relativamente frecuentes en adultos con una prevalencia en autopsias entre un 1 a un $5 \%$ de la población².
Existe una serie de factores bien conocidos como el tamaño, la forma y su localización que aumentarían su posibilidad de ruptura, teniendo en cuenta que la gran mayoría de ellos tiene una historia natural en donde el $50-80 \%$ 
no genera una hemorragia subaracnoidea $^{3,4,5}$, pese a lo cual es un problema de salud pública en Chile por su alta morbimortalidad y el elevado costo de su tratamiento.

En Chile la incidencia de la enfermedad cerebrovascular fue estudiada entre los años 2000 y 2002 por el grupo de los Drs. Pablo Lavados y Arnold Hoppe ${ }^{6}$, recogiendo datos de hemorragia subaracnoídea en donde demostró ser la $4^{a}$ causa de enfermedad cerebrovascular en Chile con un 5\% de incidencia y tener una mortalidad al mes del $40 \%$ y a los 6 meses del $46 \%$, cifras muy parecidas a otras estadísticas internacionales, con un $50 \%$ de mortalidad por esta misma entidad.

Con los avances tecnológicos relacionados a la neuroimagen, se espera que la incidencia de los aneurismas no rotos siga aumentando y con ello el requerimiento de grupos de salud dedicados al tema.

La carótida clinoidal, inicialmente denominada como $\mathrm{C} 3$, fue reconocida como la "rodilla" de la carótida interna por Fisher en estudios angiográficos en el año $1938^{7}$ y es en el año 1984 en donde este segmento de no más de 5 $\mathrm{mm}$ de longitud, es catalogado como "clinoidal" por Lasjaunias y SantoyoVazquez ${ }^{8}$. Sólo recientemente en el año 1996, Bouthillier describe los siete segmentos de la nomenclatura actual utilizando el sentido del flujo sanguíneo, dejando el segmento clinoidal o C5 confinado a la porción proximal al anillo dural distal ${ }^{9}$.

La introducción de la clinoidectomía y en especial la técnica extradural de Akira Hakuba ${ }^{10}$, la cual fuera luego modificada y popularizada en el mundo occidental por Vinko Dolenc ${ }^{11}$ (extra y subdural combinada) ha sido la llave de acceso a una serie de lesiones en la base del cráneo de origen vascular y tumoral, lo cual ha permitido resolver una serie de lesiones antes consideradas híper complejas o incluso intratables o incurables. Para este fin, no sólo es necesario un entrenamiento quirúrgico riguroso, sino que también un acabado estudio del detalle anatómico vascular, dural, óseo y neural de la región.

En Chile, un trabajo bien documentado respecto a los aneurismas paraclinoideos es el publicado por los neurocirujanos Jorge Mura, Álvaro Ruiz y Patricio Loayza ${ }^{12}$ durante el año 2000 con 10 casos satisfactoriamente resueltos en el Instituto de Neurocirugía
Asenjo, teniendo desde entonces a la fecha más de 300 aneurismas de dicha región excluidos por el primer autor (comunicación personal por Mura).

\section{Material y Método}

De un total de 73 aneurismas cerebrales resueltos como casuística personal a la fecha desde el año 2014, 7 de ellos (9\%) corresponden a aneurismas en la región paraclinoidea.

El presente trabajo corresponde a un estudio prospectivo de una serie de pacientes operados como primer cirujano en el Hospital Regional de Temuco comprendidos entre junio de 2015 y julio de 2016 (13 meses).

La serie consta de 5 pacientes y 7 aneurismas paraclinoideos del segmento oftálmico, excluyendo a 1 paciente (octavo aneurisma) por no tener registro, control ambulatorio diferido y no haber realizado clinoidectomía para la resolución de su caso.

De esta serie, 4 aneurismas requirieron resolución quirúrgica de urgencia siendo derivados por Neurorradiólogo Intervencionista luego de ser considerados poco favorables o no susceptibles para terapia endovascular. El resto se realizó en la misma paciente de manera electiva en distintos tiempos quirúrgicos por tener patología vascular proximal bilateral.

\section{Técnica quirúrgica empleada}

Anestesia general, profilaxis antibióti- ca y cabezal de Mayfield con cabeza rotada hacia contralateral y deflexión para obtener una mejor visualización de la clinoides anterior a la vez de la retracción natural del lóbulo frontal por gravedad.

Primer tiempo de exposición y disección de carótida cervical interna para preparación de clipaje transitorio mediante bulldog clamp a cargo del ayudante durante la cirugía en casos pertinentes $o$ de ruptura intraoperatoria incidental (Figura 1).

Craneotomía mini pterional con técnica interfascial subperióstica modificada de Yasargil (Figura 2). Sección de la banda meníngea orbito-temporal y fresado del ala menor del esfenoides exponiendo la fisura orbitaria superior.

Resección parcial basal del techo orbitario y de la pared lateral de la órbita respetando la grasa peri orbitaria para no agregar morbilidad y visualizar la porción intracanalicular distal del nervio óptico. Se libera de manera completa la clinoides anterior in situ, para lo cual es necesario la disección de la membrana carótido oculomotora y el "mini peeling" o peeling restringido al área en cuestión para su visualización y disección en $360^{\circ}$ y base previo a su retiro.

Se prosigue con el destechamiento del canal óptico y con la clinoidectomía anterior extradural con retiro del pilar óptico y manejo de la hemorragia venosa dependiente de la manipulación del collar carotídeo, la membrana carótido oculomotora y seno cavernoso mediante $0,5 \mathrm{cc}$ aprox de cola de fibrina Beriplast $^{\circledR}$ o mini fragmentos de Surgicel ${ }^{\circledR}$ no compresivos.

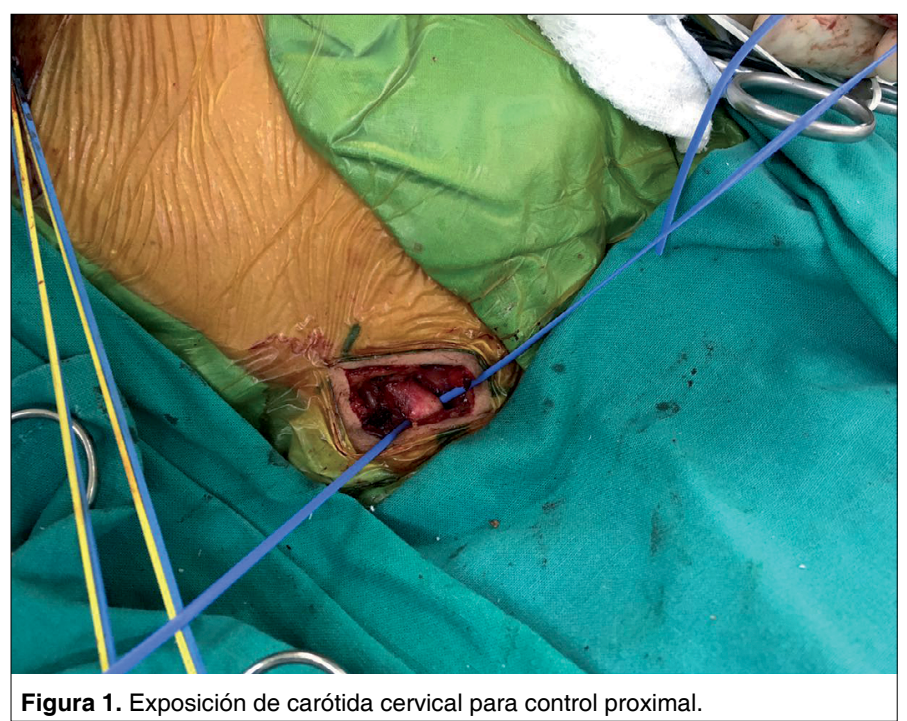




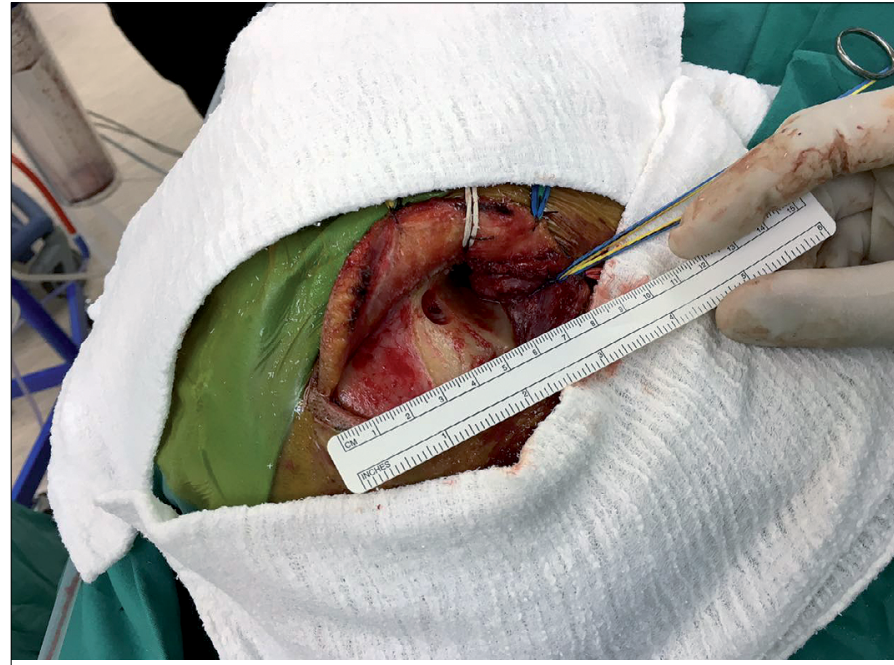

Figura 2. Craneotomía mini pterional interfascial subperióstica.

Una vez por subdural y mediante el uso de microtijera, se secciona el anillo dural distal permitiendo con esto la movilización de la carótida clinoidal y preparación de un segmento ahora intracraneal proximal al aneurisma. Se obtiene además el espacio necesario y útil para la correcta aplicación de los clip en el eje de la carótida.

Finalmente se realiza según cada caso en particular la disección vascular de la carótida clinoídea, arteria oftálmica, aneurisma cerebral en cuestión con ambas axilas y la disección neurovascular del nervio óptico habitualmente contactado y muchas veces deformado y adelgazado por el saco aneurismático contra el ligamento falciforme y su propio canal y/o contra el primer segmento de la arteria cerebral anterior, siendo por ello de regla la sección del ligamento falciforme previo a su manipulación.

La aneurismotomía con descompresión del efecto de masa y la aneurismectomía también se realizan una vez asegurado el aneurisma con los clip pertinentes, a su vez que sirve de evidencia de la exclusión del mismo.

En cuanto al cierre dural, se debe tener la precaución de cerrar el triángulo clinoidal en la base del cráneo para hermetizar la duramadre, lo cual se consigue con parche de músculo y el resto de cola de fibrina.

El uso de espátula durante la cirugía se restringe al mínimo necesario y de preferencia se utiliza el aspirador como retractor dinámico e intermitente, no siendo aconsejable su uso al inicio de la cirugía de aneurismas rotos debido a su habitual adherencia fronto basal.

\section{Presentación de los pacientes intervenidos}

1. L.M.T., mujer de 33 años, sobrepeso y fumadora, ingresa con historia de cefalea ictal y compromiso de conciencia recuperado hasta GCS 14 (WFNS II) con disartria y disminución de la agudeza visual del ojo izquierdo. Estudio con TC y angioTC de cerebro evidencia una hemorragia subaracnoídea densa con hematoma interhemisférico y hemoventrículo (mFisher 4) con hidrocefalia incipiente secundario a un aneurisma carótido oftálmico izquierdo roto de $12 \mathrm{~mm}$ de proyección superior (Figura 3a y 3b).

Por no contar con disponibilidad de neurorradiólogo intervencional en un plazo menor a 72 horas se opta por terapia quirúrgica evidenciándose gran desplazamiento del nervio óptico por parte del aneurisma y logrando su exclusión y reconstrucción de la carótida supraclinoidea mediante 2 clip en ángulo recto de cierre lateral instalados en el eje de la carótida. Se completó el procedimiento con la apertura del mismo y se continuó con el aseo y lavado cisternal además de la apertura de la membrana de Liliequist y fenestración de la lámina terminalis como es habitual en nuestro grupo en los pacientes con aneurismas rotos.

2. N.V.R., mujer de 78 años, obesa e hipertensa arterial con mal control farmacológico, ingresa por cefalea ictal y desorientación temporoespacial posterior (WFNS II). Estudio con TC y angioTC de cerebro evidencia una hemorragia subaracnoídea densa sin hemoventrículo (mFisher 2) con hidrocefalia incipiente secundaria a un aneurisma cerebral roto carótido oftálmico derecho mamelonado y grande de 15 $\mathrm{mm}$ (Figura 4a y 4b). Neurocirujano de turno deriva a terapia endovascular, no logrando resolver la patología por esta vía por falta de insumos y dificultades técnicas debido a la tortuosidad en la carótida cervical siendo derivada nuevamente a Neurocirugía.

Intraoperatoriamente se identificó un aneurisma roto complejo en su anatomía y tamaño por su cuello ancho y placas de ateroma en su base, siendo necesario para su exclusión la aplicación de la técnica de Drake con 2 clips fenestrados rectos en tándem y refuerzo con 2 clip rectos para luego descomprimir el nervio óptico severamente

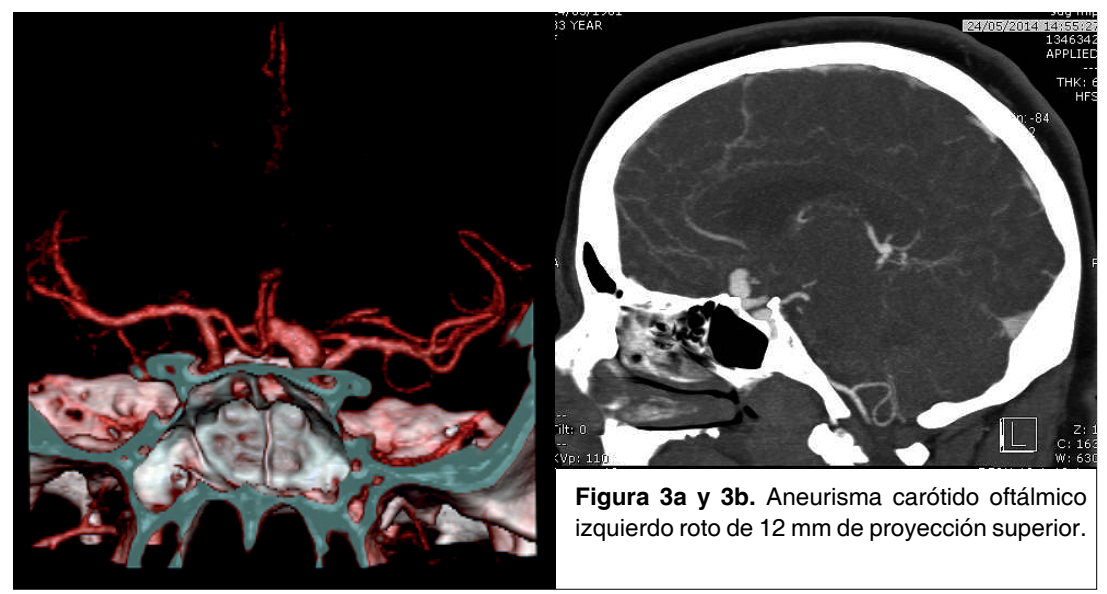




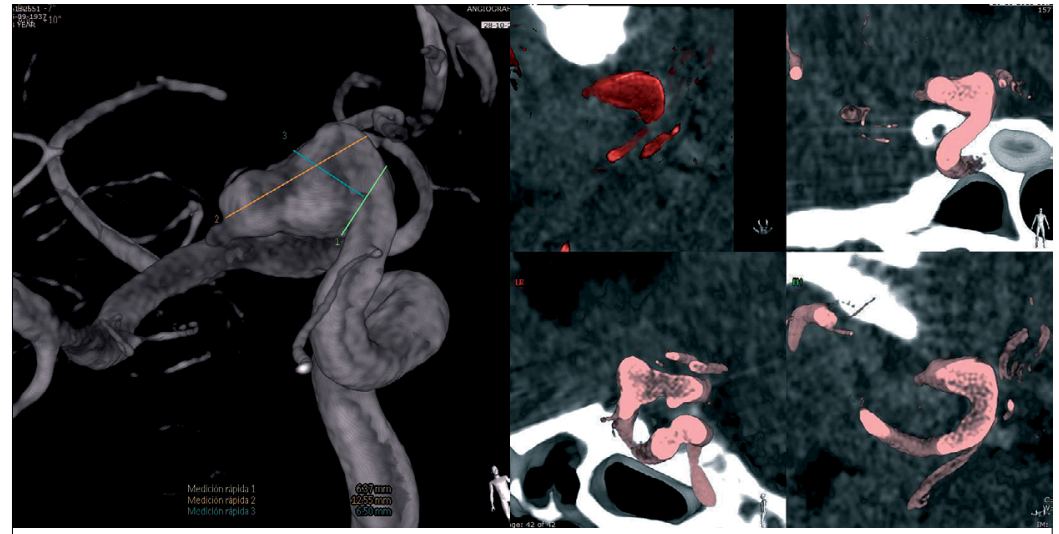

Figura 4a y 4b. Aneurisma cerebral roto carótido oftálmico derecho mamelonado y grande de $15 \mathrm{~mm}$ con placas de ateroma en su base.

desplazado y elongado hacia contralateral con aneurismotomía y exéresis del mismo.

3. E.O.M., mujer de 46 años, obesa, hipertensa arterial y con insulinoresistencia en dietoterapia quien durante septiembre de 2015 presenta trauma craneal sin compromiso de conciencia debido a accidente vehicular. Por persistencia de cefalea y mareos consulta a neurólogo quien realiza estudio con TC y luego angiografía SD de cerebro que evidencia una hemorragia subaracnoídea no densa en cisternas de la base sin claro predominio asociado a un hematoma subdural laminar hemisférico izquierdo y 4 aneurismas cerebrales interpretados como no rotos: carótido oftálmico de $10 \mathrm{~mm}$ de proyección superior izquierdo (Figura $5 a)$, aneurisma de $2,5 \mathrm{~mm}$ oftálmico verdadero derecho, aneurisma de 1,5

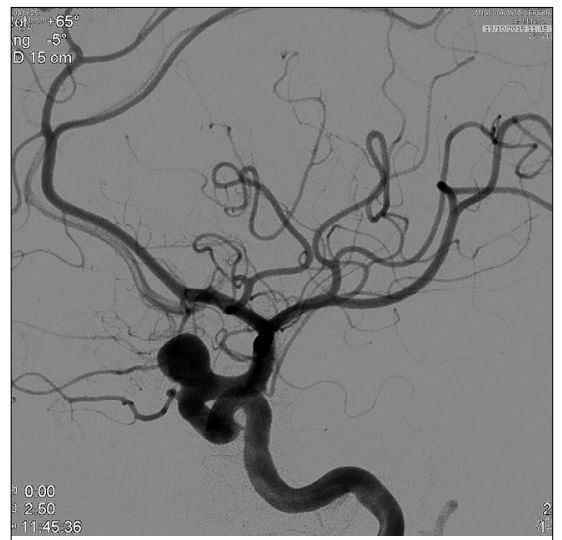

Figura 5a. Aneurisma carótido oftálmico de 10 $\mathrm{mm}$ de proyección superior izquierdo. $\mathrm{mm}$ carótido oftálmico de proyección superior derecho (Figura 5b) y aneurisma de 2,4 $\mathrm{mm}$ de bifurcación silviana derecha.

Por tener patología bilateral compleja proximal se optó por la exclusión con craneotomía bilateral. En un primer tiempo se abordó el aneurisma oftálmico izquierdo, el cual como es costumbre en los paraclinoideos grandes, ejercía efecto de masa sobre el nervio óptico destacando su delgadez y elongación. Se logró excluir con 2 clip en ángulo recto de cierre lateral aplicados en el eje de la carótida sin utilizar clipaje transitorio o trapping (Figura 5c).

El segundo tiempo quirúrgico se realiza 1 semana después, realizando apertura restringida del valle silviano derecho para la identificación y exclusión con 1 mini clip recto del baby aneurisma de bifurcación silviana. Se procede luego a la exclusión del aneurisma depen-

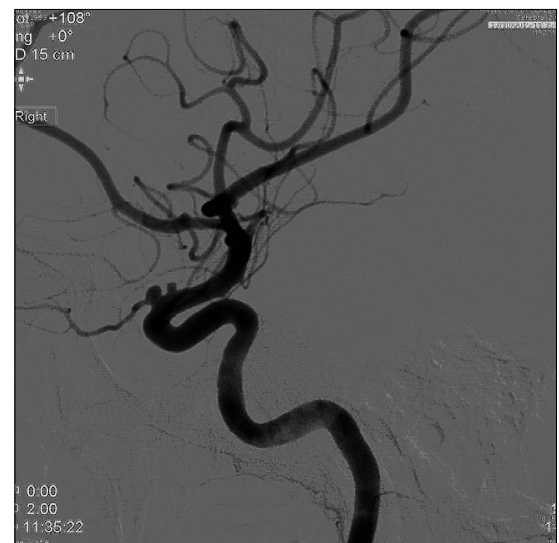

Figura 5b. Aneurismas a derecha de $2.5 \mathrm{~mm}$ oftálmico verdadero y de $1,5 \mathrm{~mm}$ carótido oftálmico de proyección superior. diente de la arteria oftálmica, para lo cual es necesario disecarlo del nervio óptico en el cual parecía estar embebido y bajar el flujo sanguíneo carotídeo mediante el clipaje transitorio ultra corto sin trapping y dejar ex professo un mínimo de remanente aneurismático que permitiera la salida de la arteria oftálmica sin acodamiento ni estenosis de la misma, reforzando la pared con un fragmento pequeño de fascia temporal y aplicación de 1 mini clip recto. Se excluye por último el aneurisma oftálmico de proyección superior algo más distal al previo sobre la carótida dorsal con 1 mini clip recto (Figura $5 d$ ). Destaca la insospechada dificultad en el clipaje del aneurisma oftálmico verdadero secundario al alto flujo y la reiterada expulsión del clip antes del uso de clipaje transitorio.

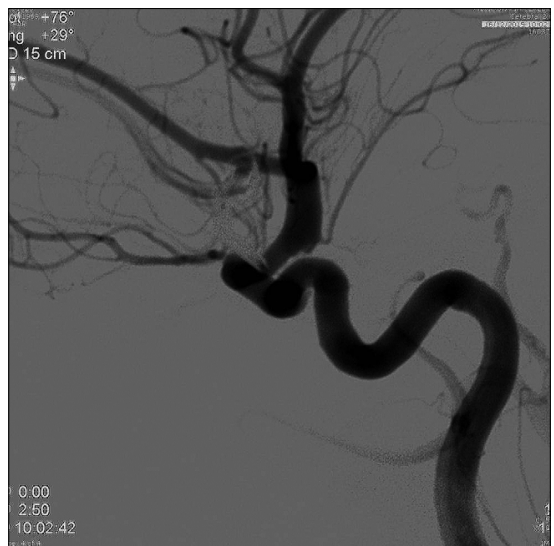

Figura 5c. Exclusión de aneurisma izquierdo con 2 clip en ángulo recto de cierre lateral aplicados en el eje de la carótida.

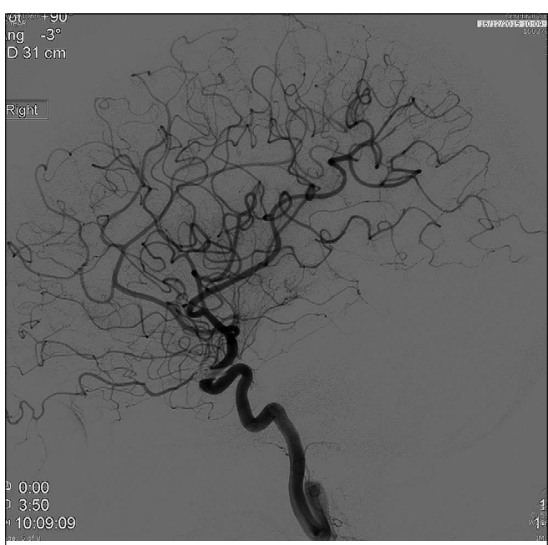

Figura 5d. Exclusión de aneurismas a derecha utilizando mini clip y dejando ex professo un mínimo remanente que permitiera la salida de la arteria oftálmica sin acodamiento ni estenosis de la misma. 


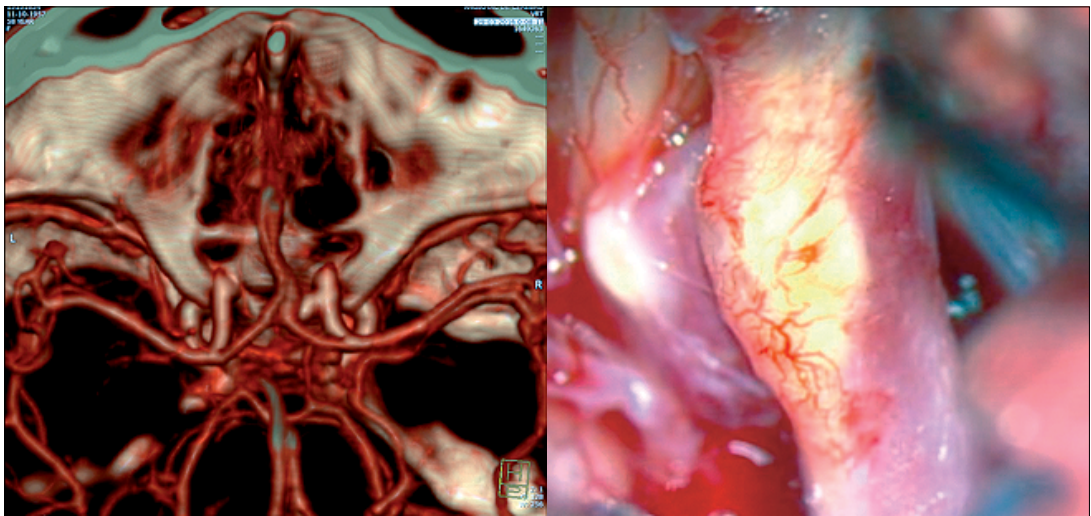

Figura $\mathbf{6 a}$ y $\mathbf{6 b}$. Aneurisma roto paraclinoideo de pared medial de $6 \mathrm{~mm}$ mamelonado que desplaza al nervio óptico.

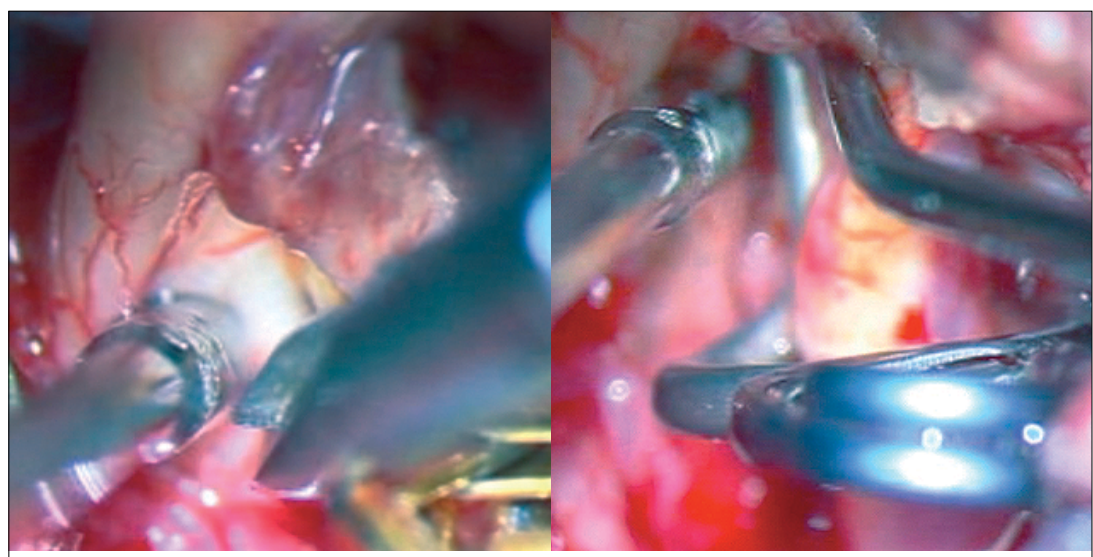

Figura $6 \mathrm{c}$ y $6 \mathrm{~d}$. Microdisección con tijera y reconstrucción carotídea con clip fenestrado en ángulo recto.

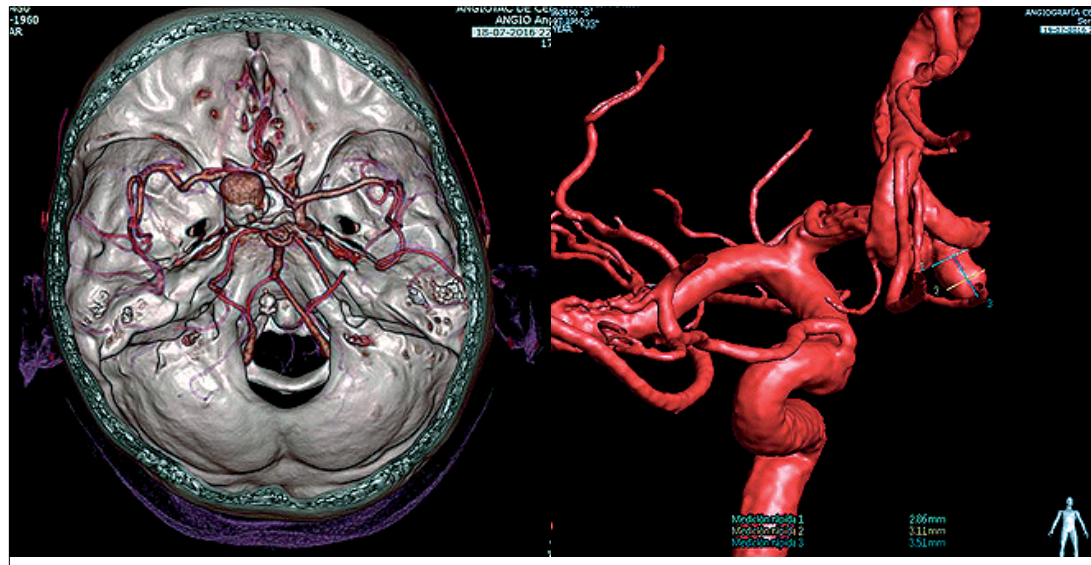

Figura $7 \mathbf{a}$ y $\mathbf{7 b}$. Aneurisma roto paraclinoideo de fusiforme dirigido hacia ventral de $17,17 \mathrm{~mm}$ de diámetro mayor calcificado y parcialmente trombosado en su domo con mamelón de ruptura y aneurisma comunicante anterior dirigido hacia izquierda de $3,5 \mathrm{~mm}$
4. E.S.T., mujer de 58 años, sobrepeso e hipertensa arterial ingresa por cefalea ictal con compromiso de conciencia evidencian una hemorragia subaracnoídea densa de predominio basal derecho sin hemoventrículo (mFisher 3 ), pero con incipiente hidrocefalia secundaria a un aneurisma roto paraclinoideo de pared medial de $6 \mathrm{~mm}$ mamelonado (Figura 6a y 6b).

El aneurisma tenía cuello ancho y levantaba al nervio óptico ipsilateral requiriendo una meticulosa disección del plano nervio óptico-aneurisma con aneurismectomía previo a su exclusión y mayor apertura del anillo dural distal. La disección carotídea generó el espacio necesario para la instalación de un clip transitorio intracraneal post oftálmica y pre aneurisma hasta su reconstrucción y exclusión aneurismática mediante el uso de un clip fenestrado en ángulo recto de $5 \mathrm{~mm}$ (Figura 6c y 6d).

5. A.E.R., mujer de 55 años, hipertensa arterial con mal manejo de presiones tensionales es llevada a urgencia en GCS 12 con hemiplejia derecha (WFNS IV) luego de haber presentado cefalea ictal. Estudio con TC y angioTC de cerebro evidencian una hemorragia subaracnoídea densa de predominio interhemisférico y carotídeo izquierdo con hemoventrículo (mFisher 4) sin hidrocefalia. Estudio vascular invasivo pesquisa un aneurisma roto paraclinoideo de aspecto fusiforme dirigido hacia ventral de $17,17 \mathrm{~mm}$ de diámetro mayor calcificado y parcialmente trombosado en su domo con mamelón de ruptura. Además presenta un baby aneurisma de $\mathrm{A} 1$ izquierda de $1,73 \mathrm{~mm}$, un baby aneurisma coroideo contralateral de $1,3 \mathrm{~mm}$ y un aneurisma comunicante anterior dirigido hacia izquierda de 3,5 $\mathrm{mm}$ (Figura 7a y 7b).

La terapia endovascular concluyó una mala tolerancia al test de oclusión con balón y la no susceptibilidad a la exclusión aneurismática por este método en la fase aguda (Figura 7c y 7d).

Se discutió en grupo de neurocirugía y con neurorradiólogo intervencional y se decidió en conjunto con la familia ofrecer terapia quirúrgica con bypass cerebral transitorio durante la eventual trombectomía y reconstrucción de la carótida supraclinoidea mediante fenestración.

Se llevó a cabo en primera instancia la disección de la arterial temporal superficial en su rama parietal por ser esta la de mayor calibre. Luego se continuó con la exposición cervical y la craneotomía pterional con clinoidectomía extra- hasta GCS 13 (WFNS II) e intensa cefalea refractaria a terapia analgésica. Estudio con TC y angioTC de cerebro 

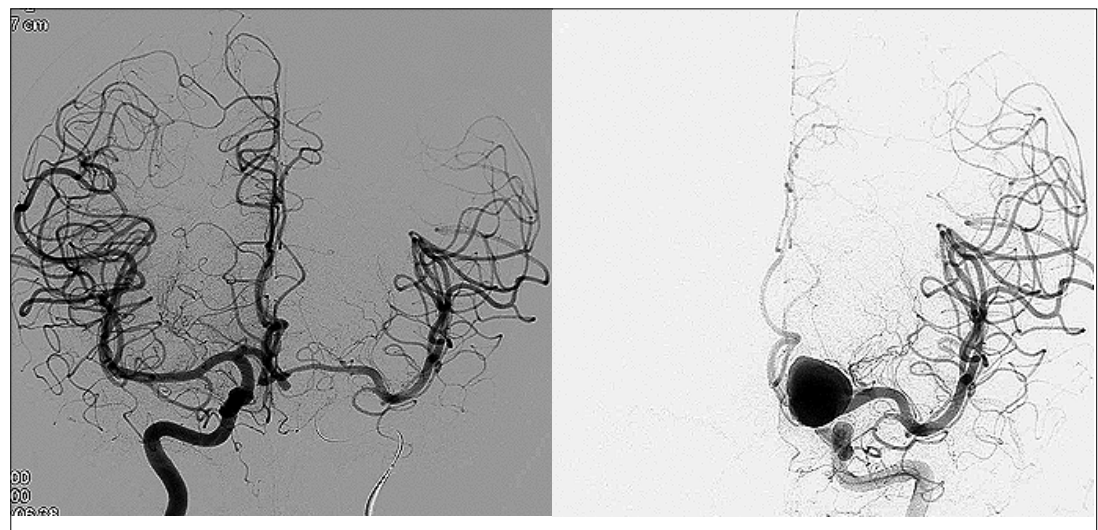

Figura 7c y 7d. Mala tolerancia al test de oclusión con balón.

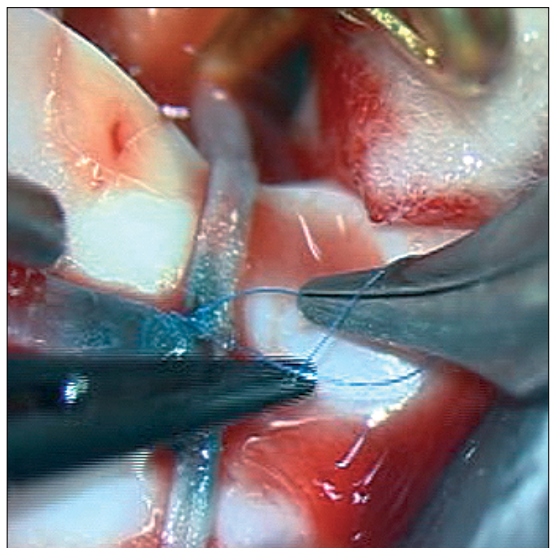

Figura 7e. Bypass STA/MCA con sutura $9 / 0$ puntos separados utilizando sección oblicua y "fishmouth".

dural al igual que el resto de los casos descritos, destacando aquí la amplia apertura del valle silviano y la elección de una rama temporal de similar calibre a la temporal superficial seleccionada. Se realizó un bypass término lateral STA/MCA izquierdo con sutura 9/0 puntos separados utilizando sección oblicua y "fishmouth" en la rama temporal para aumentar su diámetro y obtener un flujo más oblicuo (Figura 7e). Se comprobó la permeabilidad del bypass de forma visual y se continuó luego con la disección del aneurisma paraclinoideo el cual se objetiva muy grande y proximal, requiriendo una apertura del anillo dural completa en pro de permitir la visualización de la carótida proximal extracraneal sana y disecar el cuello proximal (Figura 7f).

Luego de la disección del aneurisma del nervio óptico, del oculomotor y del seno cavernoso, se concluye que es imposible la reconstrucción con clip por

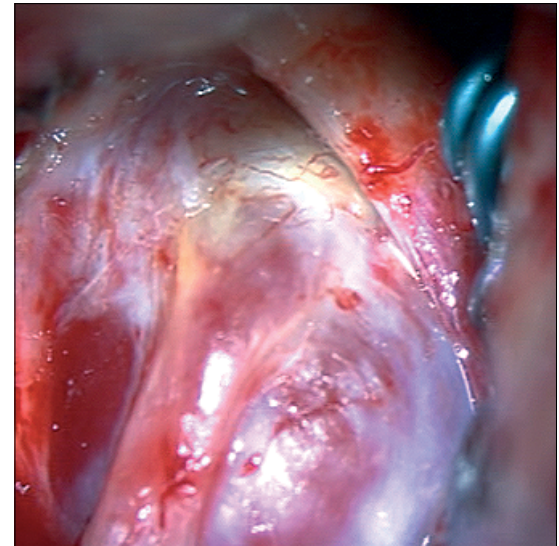

Figura 7f. Aneurisma fusiforme amorfo sin pared arterial sana que permita su fenestración.

corresponder su anatomía a un aneurisma fusiforme amorfo y no presentar pared arterial sana que permita una fenestración carotídea. Se explora el resto de los aneurismas (A1 y comunicante anterior) y se excluyen con clip, momento en el cual se produce ruptura intraoperatoria profusa de muy difícil control y que obliga a realizar trapping del aneurisma dejando permeable tanto la arteria oftálmica como la coroidea, asumiendo ahora el bypass como definitivo a modo de aumentar el flujo dependiente desde la arteria comunicante anterior reparada (Figura $7 \mathrm{~g}$ ).

\section{Resultados}

La incisión en piel y craneotomías fueron restringidas en su tamaño logrando así evitar edema postoperatorio y complicaciones del cierre. Además, al haber hecho una resección parcial y basal del techo orbitario y pared lateral de la órbita con respeto de la grasa peri orbitaria, no se registró edema importante o dolor peri orbitario, oscilopsia ni parálisis del elevador del párpado.

La exposición cervical tuvo un tiempo promedio de 16 minutos y la clinoidectomía de otros 32 .

Los tiempos neuroquirúrgicos (incluyendo la exposición cervical) se presentan ordenados en la Tabla 1. En ella destaca que existe una demora significativamente mayor en la resolución de los casos con aneurisma cerebral roto atribuibles a la dificultad en la disección aracnoidal, al aseo cisternal y al lavado a través de la lámina terminalis fenestrada y membrana de Liliequist abierta a modo de contribuir en la terapia contra la hidrocefalia y el vasoespasmo clínico, destacando que en estos, como en otros aneurismas rotos aunque de otras localizaciones operados por el mismo grupo, no han requerido uso de drenaje ventricular externo, derivación ventrículo peritoneal ni han tenido tampoco deterioro neurológico atribuido a vasoespasmo.

En los aneurismas con antecedente de ruptura, se realizó clipaje transitorio cervical menor a 5 minutos con trapping previo a su manipulación y final exclusión con clips.

No hubo requerimiento de transfusión sanguínea y pudieron continuar su post operatorio en UCI (casos rotos) o UTI (casos no rotos) en donde se les realizó angioTC de cerebro de control antes de 24 horas.

En la Tabla 2 se resume por cada paciente su condición clínica de ingreso, las características de los aneurismas y

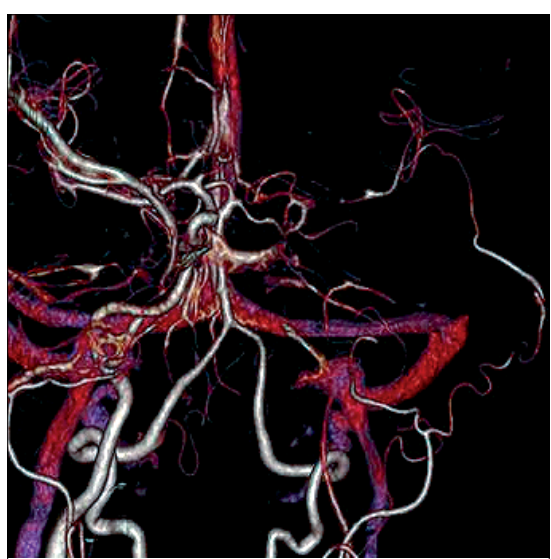

Figura 7g. Reconstrucción final mostrando bypass STA/MCA con flujo insuficiente a izquierda. 
Tabla 1

\begin{tabular}{|l|l|c|}
\hline Nombre & Localización & Tiempo operatorio (min) \\
\hline L.M.T. & Oftálmico roto & 300 \\
\hline N.V.R. & Oftálmico roto & 300 \\
\hline \multirow{2}{*}{ E.O.M. } & Oftálmico no roto izquierdo & 240 \\
\cline { 2 - 3 } & $\begin{array}{l}\text { Oftálmico verdadero no roto derecho + Oftálmico no roto derecho + Baby } \\
\text { silviano derecho no roto }\end{array}$ & 300 \\
\hline E.S.T. & Hipofisiario roto & 300 \\
\hline A.E.R. & Oftálmico roto trombosado y calcificado & 360 \\
& & $(30$ minutos de anastomosis) \\
\hline
\end{tabular}

\begin{tabular}{|c|c|c|c|c|c|c|c|c|}
\hline Nombre & Edad & Sexo & Localización & $\begin{array}{l}\text { Tamaño } \\
(\mathrm{mm})\end{array}$ & WFNS & FISHER & Complicación & Resultado \\
\hline L.M.T. & 33 & $\mathrm{~F}$ & Oftálmico roto & 12 & 2 & 4 & - & Indemne \\
\hline N.V.R. & 78 & $\mathrm{~F}$ & Oftálmico roto & 15 & 2 & 2 & - & Indemne \\
\hline \multirow[t]{4}{*}{ E.O.M. } & \multirow[t]{4}{*}{46} & \multirow[t]{4}{*}{$\mathrm{F}$} & Oftálmico no roto & 10 & - & - & Ventriculitis & Indemne \\
\hline & & & $\begin{array}{l}\text { Oftálmico verda- } \\
\text { dero no roto }\end{array}$ & 2,5 & & & & \\
\hline & & & Oftálmico no roto & 1,5 & & & & \\
\hline & & & Baby silviano & 2,4 & & & & \\
\hline E.S.T. & 58 & $\mathrm{~F}$ & Hipofisiario roto & 6 & 2 & 3 & - & Indemne \\
\hline A.E.R. & 55 & $\mathrm{~F}$ & $\begin{array}{l}\text { Oftálmico roto } \\
\text { trombosado y } \\
\text { calcificado }\end{array}$ & 17 & 4 & 4 & $\begin{array}{l}\text { Trapping de } \\
\text { carótida no } \\
\text { tolerante }\end{array}$ & Fallece \\
\hline
\end{tabular}

su resultado clínico final.

La tercera paciente intervenida evolucionó con ventriculitis por Pseudomona aeruginosa requiriendo antibioterapia prolongada que no trajo repercusión clínica.

Análisis especial requiere la última paciente en quien se realizó bypass cerebral, siendo llevada a AngioTC cerebral post operatorio en donde se objetiva la exclusión de los aneurismas y la patencia del bypass cerebral además de descartar complicaciones hemorrágicas o isquémicas. Continuó su postoperatorio sedada en $\mathrm{UCl}$ en donde cursó con neumonía aspirativa y agravó su condición de disnatremia llegando a tener un sodio plasmático de 180 $\mathrm{mEq} / \mathrm{l}$ durante las horas inmediatas a la cirugía y falleciendo el día 12 de la hemorragia subaracnoídea durante su recuperación en $\mathrm{UCI}$ sin poder obtener un TC de cerebro diferido a solicitud de familiares de limitar todo esfuerzo terapéutico.

En controles ambulatorios y como es habitual en casos con hemorragia subaracnoídea, las primeras dos pacientes relataron cefalea crónica inespecífica y molestias vagas en relación a la craneotomía, lo cual cedió completamente al tercer mes mediante el uso de AINEs en caso necesario.

No hubo deterioro cognitivo y no se agregó daño neurológico o deterioro visual, llegando incluso a resolución temprana de estas molestias con mejoría del campo visual relatada en forma subjetiva al no contar en el Hospital Regional de Temuco con acceso fluido a estudio oftalmológico previo para efectos de comparación y estudio.

No hubo fístula de líquido cefalorraquídeo, atrofia del músculo temporal o menoscabo estético craneal o cervical. Todos han sido controlados en forma ambulatoria con estudio de angioTC y/o angiografía SD que ha demostrado la exclusión aneurismática sin requerimiento de nuevos procedimientos siendo dados de alta de sus controles y retornando a sus labores habituales previa hospitalización sin terapia farmacológica alguna.

\section{Conclusión y Discusión}

Los aneurismas de la región clinoidal y proximales al origen de la arteria comunicante posterior generalmente son de morfología irregular y $>7 \mathrm{~mm}$, lo cual conlleva un alto riesgo de ruptura en el tiempo ${ }^{13}$. En el caso de los pacientes presentados con aneurismas rotos, estos tienen en común el género femenino, la hipertensión arterial y el sobrepeso como factores que pudiesen correlacionarse con el riesgo de ruptura de aneurismas cerebrales en forma independiente del tamaño ${ }^{14}$.

Los aneurismas en relación a la clinoides representan alrededor del $10 \%$ del total y si bien el número de pacientes seleccionados para este trabajo es reducido, este corresponde a una serie personal inicial y a un contexto regional en donde lo habitual era la resolución 
por vía endovascular o su derivación a otro centro, contribuyendo a esto último la complejidad anatómica y neuroquirúrgica de dichos aneurismas, lo laborioso en obtener control proximal y exposición completa del saco y sus axilas, el habitual desconocimiento en el manejo de disección y exposición de la carótida cervical por parte de algunos neurocirujanos y también al desconocimiento de la herramienta quirúrgica por parte de médicos generales, neurólogos e incluso de algunos cirujanos cerebrales.

Es importante mencionar, que si bien no es objeto de discusión en el presente trabajo, la última evidencia disponible gracias al reporte del estudio BRAT a los 6 años ${ }^{15}$ establece que la tasa de obliteración aneurismática es mayor para la opción quirúrgica ( $96 \%$ vs $48 \%$ ) y tanto el retratamiento $(4,6 \%$ vs $16,4 \%)$ como el traspaso de pacientes a la otra terapia (1,9\% vs 38\%) favorece al clip. Esto no se tradujo en mayores eventos hemorrágicos o de muertes en el grupo tratado con coils y vino a contribuir a la discusión sobre la superioridad o no de una terapia sobre otra. Pienso que debe entenderse como una reafirmación al hecho de la necesidad de la coexistencia de ambas y que las dos son técnicas validadas y duraderas según sean las características del aneurisma, del paciente y la expertise de cada grupo, para así ofrecer lo mejor según sea el caso y el contexto.

La neurocirugía ha ido buscando nuevas técnicas y accesos quirúrgicos que también permitan acortar los tiempos operatorios y exposición cerebral minimizando las posibles complicaciones. En este sentido sobresalen las técnicas mediante accesos Keyhole de Dr. Perneczki y la evolución de la craneotomía pterional clásica de Yasargil a la mini pterional de Nathal y Figueiredo ${ }^{16,17}$, las cuales pueden ser utilizadas en cirugías tan complejas como estas.

En cuanto a la inflamación vascular y fibrosis leptomengínea, se sabe que tienen directa relación con la fisiopatología del déficit neurológico isquémico tardío e hidrocefalia que requiere derivación en los casos de hemorragia subaracnoídea densa ${ }^{18}$. Es así como el esfuerzo neuroquirúrgico se ha vuelto protocolizado en muchas partes del mundo y adoptado en nuestro servicio con el clearence cisternal de coágulos, apertura de la membrana de Liliequist conectando la región supra con la infratentorial y fenestrando la lámina terminalis sin utilizar drenaje ventricular externo intraoperatorio o en algún momento de la fase aguda para este fin.

En los casos descritos la terapia quirúrgica fue pronta en los tiempos de resolución y óptima elección en los casos de aneurismas rotos, ya que como en los casos presentados, es habitual que estos aneurismas sean grandes y tengan cuello ancho no siendo susceptibles a terapia endovascular. Últimamente se ha ido resolviendo dicho factor mediante el uso de diversores de flujo con doble antiagregación posterior, no siendo recomendable en los casos de hemorragia subaracnoídea y con indicaciones restringidas por preocupaciones sobre su seguridad hasta la fecha.

Atingente a la paciente fallecida, un escenario quirúrgico ideal y programado con el aneurisma no roto, hubiese incluido estudio vascular periférico para preparación de una arteria radial por un cirujano vascular y luego bypass de alto flujo para trapping del aneurisma con reemplazo del flujo carotídeo. Si bien la paciente intervenida falleció pese a la patencia del bypass realizado y que muy posiblemente su flujo era insuficiente a pesar de sumarse al de comunicante anterior, dicha terapia representa para nosotros como grupo una nueva herramienta útil en patologías complejas como la de esta persona y en otras afecciones como la isquemia crónica, tumores cerebrales y reparaciones vasculares iatrogénicas. Muchos neurocirujanos hemos participado del programa de formación complementaria en Chile para perfeccionamiento en técnicas de neurocirugía vascular y de la base del cráneo, lo cual ha permitido lograr un mayor conocimiento y replicar una serie de técnicas en estas materias, haciéndolas de uso común en la neurocirugía cerebral moderna en el país y logrando así participar en distintos centros de provincia como Temuco, el cual pertenece a los 8 hospitales catalogados como centro A, sinónimo de su alta complejidad y un amplio compromiso de parte del grupo para resolver patologías. La cohesión del grupo en Temuco (neurocirujanos, neurorradiólogo intervencional, anestesistas, enfermeras y arsenaleros) es de destacar y se ve reflejada en las extensas reuniones y discusiones que intentan ofrecer lo mejor para todos los pacientes tratados.

Como falencias locales es no contar con vídeo angiografía intraoperatoria con verde de indocianina (ICG por sus siglas en inglés) en el Hospital Regional de Temuco, lo cual es una herramienta no invasiva, rápida, dinámica, de fácil aplicación y muy útil en demostrar la exclusión completa del aneurisma y la permeabilidad de vasos adyacentes como las perforantes ${ }^{19,20}$. Tampoco disponemos de doppler cerebral intraoperatorio o flujómetro, el cual permite interpretar el cambio en la velocidad de flujo y conocer estenosis $\mathrm{u}$ oclusiones adquiridas por el procedimiento además de la patencia de puentes arteriales.

Las distintas técnicas microquirúrgicas existentes en la patología vascular y de la base del cráneo, son capaces de ofrecer en un mismo acto quirúrgico, la resolución de múltiples aneurismas logrando su exclusión y junto a ello, la descompresión neurovascular de los nervios comprometidos en forma uni o bilateral además del lavado cisternal y de la fenestración de la lámina terminalis tan útil en el manejo de la hidrocefalia y en la batalla contra el vasoespasmo clínico.

Las habilidades neuroquirúrgicas son junto al conocimiento, el armamentarium de todo neurocirujano, siendo estas destrezas de vital importancia para la resolución de patologías cada vez más complejas a las que nos vemos enfrentados, pero que sin embargo, seguiremos jugando un rol fundamental en la resolución de estos casos tanto de urgencia, como electivos, logrando a futuro reducir tiempos anestésicos y quirúrgicos y por sobre todo, dar a conocer en la comunidad médica la resolución neuroquirúrgica de los aneurismas paraclinoideos con buenos resultados clínicos para los pacientes y un bajo costo para las instituciones al ser una terapia sin recursos extraordinarios, ampliamente demostrada en su utilidad y por lo general, única y definitiva.

Recibido: 03 de marzo de 2017 Aceptado: 15 de abril de 2017 


\section{Referencias}

1. República de Chile, Ministerio de Salud 2007. Guía Clínica Hemorragia Subaracnoidea Secundaria a Rotura de Aneurismas Cerebrales.

2. Wiebers DO, Whisnant JP, Huston J III, et al. Unruptured intracranial aneurysms: natural history, clinical outcome, and risks of surgical and endovascular treatment. Lancet 2003; 362: 103-10.

3. Go AS, Mozaffarian D, Roger VL, et al. On behalf of the American Heart Association Statistics Committee and Stroke Statistics Subcommittee. Heart disease and stroke statistics - 2014 update: a report from the American Heart Association. Circulation 2014; 129: e28-e292. doi: 10.1161/01. cir.0000441139.02102.80.

4. Steiner T, Juvela S, Unterberg A, Jung C, Forsting M, Rinkel G. European Stroke Organization. European Stroke Organization guidelines for the management of intracranial aneurysms and subarachnoid haemorrhage. Cerebrovasc Dis. 2013;35:93-112. doi: $10.1159 / 000346087$.

5. Rinkel GJ, Djibuti M, Algra A, van Gijn J. Prevalence and risk of rupture of intracranial aneurysms: a systematic review. Stroke. 1998; 29: 251-256.

6. Lavados PM, Sacks C, Prina L, et al. Incidence, 30-day case-fatality rate, and prognosis of stroke in lquique, Chile: a 2-year community based prospective study (PISCIS project). Lancet 2005; 365: 2206-2215.

7. Fischer E. Die Lageabweichungen der vorderen Hirnarterie im Gefäßbild. Zentralbl Neurochir 1938; 3: 300-313.

8. Lasjaunias P, Santoyo-Vazquez A. Segmental agenesis of the internal carotid artery: Angiographic aspects with embryological discussion. Anat Clin 1984; 6: 133-141.

9. Bouthillier A, van Loveren HR, Keller JT. Segments of the internal carotid artery: A new classification. Neurosurgery 1996: $38: 425-433$.

10. Hakuba A, Nishimura S, Shirakata S, Tsukamoto M. Surgical Approaches to the Cavernous Sinus. Neurol med chir. Tokio. 1982; 22: 295-308.

11. Dolenc VV. A combined epi-and subdural direct approach to carotid-ophthalmic artery aneurysms. J Neurosurg 1985; 62: 667-672.

12. Mura J, Ruiz A, Loayza P. Manejo neuroquirúrgico de los aneurismas paraclinoideos. Rev Chil Neuro-Psiquiat 2000; 38: 268-291.

13. The Natural Course of Unruptured Cerebral Aneurysms (UCAS) in a Japanese Cohort. N Engl J Med 2012; 366: $2474-2482$.

14. Small Unruptured Intracranial Aneurysm Verification Study (SUAVe Study). Stroke 2010, 41: 1969-1977.

15. The Barrow Rupture Aneurysm Trial (BRAT): 6-year results. J Neurosurg 2015; 123: 609-617.

16. Nathal E, Gómez-Amador JL. Anatomic and surgical basis of the sphenoid ridge keyhole approach for cerebral aneurysms. Neurosurgery 2005; 56: 178-185.

17. Figueiredo E, Deshmukh P, Nakaji P, et al. The minipterional craniotomy: technical description and anatomic assessment. J Neurosurg. 2007; 61: 256-265.

18. Mura J, et al. Improved Outcome in High-Grade Aneurysmal Subarachnoid Hemorrhage by Enhancement of Endogenous Clearance of Cisternal Blood Clots: A Prospective Study that Demonstrates the Role of Lamina Terminalis Fenestration Combined with Modern Microsurgical Cisternal Blood Evacuation. Minim Invas Neurosurg 2007; 50: 355-362.

19. Chiang VL, Gailloud P, Murphy KJ, Rigamonti D, Tamargo RJ. Routine intraoperative angiography during aneurysm surgery. J Neurosurg 2002; 96: 979-980.

20. De Oliveira JG, Beck J, Seifert V, Texeira MJ, Raabe A. Assessment of flow in perforating arteries during intracranial aneurysm surgery using intraoperative near-infrared indocyanine green videoangiography. Neurosurgery 2007; 61: ONS-63-ONS-73.

\section{Correspondencia a:}

Dr. Oscar Jiménez Palma

Neurocirugía, Hospital Regional de Temuco.

oscarjimenezpalma@gmail.com 